\title{
Vascular neurocutaneous disorders: neurospinal and craniofacial imaging findings
}

\author{
Ahmed Abdel Khalek Abdel Razek
}

Received: 10 April 2014/ Accepted: 8 July 2014/Published online: 31 July 2014

(C) Japan Radiological Society 2014

\begin{abstract}
We review the neurospinal and craniofacial imaging findings in vascular neurocutaneous disorders. The patients presented with cutaneous and cerebral lesions associated with craniofacial abnormalities or spinal lesions. Vascular neurocutaneous disorders may involve segmental or localized lesions associated with either low- or highflow vascular malformations. Other neuroimaging findings include vascular occlusive changes with ischemic stroke, ectatic arteries, aneurysm, cortical migrational disorders such as hemimegalencephaly and congenital anomalies of the posterior fossa. Craniofacial vascular malformations, eye abnormalities, facial deformity and spinal lesions have been reported in some cases. Hamartomatous formation and malignancy have also been reported in some cases. Correlation of clinical findings with neurospinal and craniofacial abnormalities is important to reach a specific diagnosis of some vascular neurocutaneous disorders.
\end{abstract}

Keywords Vascular - Malformation - Neurocutaneous . Disorder · Spinal

\section{Introduction}

The neurocutaneous disorders are a broad heterogeneous group of congenital disorders with diverse genetic, clinical and pathological features that share common developmental lesions of the skin and of the central and peripheral nervous systems [1-3]. Vascular neurocutaneous disorders are characterized as a predominant disorder of the

A. A. K. A. Razek ( $\bowtie)$

Department of Diagnostic Radiology, Mansoura Faculty of Medicine, Mansoura 13351, Egypt

e-mail: arazek@mans.edu.eg cerebrovascular system with secondary neuroectodermal changes. These disorders may be segmental, involve a large region or be a localized lesion. Segmental vascular neurocutaneous disorders include Sturge-Weber syndrome (SWS), PHACE syndrome, craniofacial arteriovenous metameric syndrome (CAMS) and spinal arteriovenous metameric syndrome (SAMS). Localized vascular neurocutaneous disorders include hereditary hemorrhagic telangiectasia (HHT), blue rubber bleb nevus syndrome (BRBNS), Klippel-Trenaunay syndrome (KTS), macrocephaly-capillary malformation (M-CM), capillary malformation-arteriovenous malformation (CM-AVM), meningioangiomatosis (MA) and ataxia-telangiectasia (AT) [1-5] (Table 1). Vascular neurocutaneous disorders manifest with high-flow, low-flow or capillary stain vascular malformations (Table 2). These disorders may be associated with cortical malformation, hemimegalencephaly, craniofacial or spinal abnormalities, and overgrowth in one region or one side of the body [4-8]. Cross-sectional imaging with CT or MR imaging is commonly used for the initial diagnosis of vascular neurocutaneous disorders; however, vascular imaging with contrast MR angiography, CT angiography and digital subtraction angiography has been used for better characterization of the associated vascular lesions $[2-6,9,10]$.

The aim of this work is to review the neurospinal and craniofacial imaging findings in vascular neurocutaneous disorders.

\section{Sturge-Weber syndrome}

Sturge-Weber syndrome (SWS), or encephalotrigeminal angiomatosis, is a nonfamilial disease with unilateral facial angioma (port-wine stain) localized in the first and less 
Table 1 Classification of vascular neurocutaneous disorders

\begin{tabular}{ll}
\hline Segmental & Localized \\
\hline Sturge-Weber syndrome & $\begin{array}{c}\text { Hereditary hemorrhagic } \\
\text { telangiectasia }\end{array}$ \\
PHACE syndrome & Blue rubber bleb nevus syndrome \\
Craniofacial arteriovenous & Klippel-Trenaunay syndrome \\
metameric syndrome & Macrocephaly-capillary \\
Spinal arteriovenous metameric & malformation \\
syndrome & Capillary malformation- \\
& arteriovenous malformation \\
& Parkes Weber syndrome \\
& Meningioangiomatosis \\
& Ataxia-telangiectasia \\
\hline
\end{tabular}

Table 2 Types of vascular malformations with vascular neurocutaneous disorders

\begin{tabular}{ll}
$\begin{array}{l}\text { Vascular } \\
\text { malformations }\end{array}$ & Vascular neurocutaneous disorders \\
\hline $\begin{array}{l}\text { Arteriovenous } \\
\text { malformation }\end{array}$ & $\begin{array}{l}\text { Craniofacial arteriovenous metameric } \\
\text { syndrome } \\
\text { Spinal arteriovenous metameric syndrome } \\
\text { Capillary malformation-arteriovenous } \\
\text { malformation } \\
\text { Hereditary hemorrhagic telangiectasia }\end{array}$ \\
Klippel-Trenaunay syndrome \\
\\
$\begin{array}{l}\text { Macrocephaly-capillary malformation } \\
\text { Capillary malformation-arteriovenous } \\
\text { malformation }\end{array}$ \\
Ataxia telangiectasia \\
Sturge-Weber syndrome \\
Blue rubber bleb nevus
\end{tabular}

often in the second and third sensory distribution of the trigeminal nerve, occasionally involving the neck and any portion of the face associated with a calcified leptomeningeal venous malformation (angioma) of the ipsilateral parieto-occipital region. SWS appears to have no predilection for any particular race or gender. Symptoms appear before the 2 nd year of age and include cosmetic and neurological problems such as epilepsy, deficits and mental retardation. Port-wine stains, which represent localized dermal venular malformations, are classically present. This facial vascular malformation usually is unilateral, but it can involve the midline and may extend to the chest, trunk and limbs $[11,12]$.

The typical CT and MRI features seen in SWS include hemi-atrophy of the cerebral hemispheres and ipsilateral leptomeningeal angiomatosis seen as vascular contrast enhancement. Prominent ipsilateral choroid plexus in the lateral ventricle and enlargement of the transmedullary venous drainage are reported in this disorder. Ipsilateral calcification is better visualized on CT scans and susceptibility weighted MR imaging. Because of the capillaryvenous vascular malformations, the cortical and white matter perfusion is altered in SWS, the degree of which is related to epilepsy. Severe cortical calcification in the affected hemisphere is related to decreased parenchymal perfusion and associated with more severe epilepsy in patients with SWS. It may be associated with ipsilateral hypertrophy of the skull and sinuses. MR imaging performed early during the course of the disease demonstrates normal or even accelerated myelination in the centrum semiovale in the involved hemisphere. Accelerated myelination is reported in infants with SWS, whereby the affected hemisphere shows areas of abnormal T2-weighted imaging and FLAIR hypointensities in the white matter. In older patients, white matter damage and loss in the affected hemisphere are seen, causing T2-hyperintense changes [1, 4, 12] (Fig. 1). Advanced MR imaging can detect microstructural changes in the cortex and white matter at an early stage of SWS when interventions can prevent irreversible changes. Deep venous collaterals are detected by susceptibility-weighted imaging, and they are often located directly adjacent to severely damaged cortical regions. Diffusion abnormalities in the white matter extend beyond cortical involvement, encompassing white matter with venous collaterals. Diffusion tensor imaging studies have reported reduced fractional anisotropy measures in both the affected and contralateral hemisphere, even in children, suggesting early onset of microstructural diffuse white matter damage. Tram-track or railroad-track calcification of the adjacent sulci described on the skull radiograph is of great significance $[11,12]$.

SWS can be subdivided into type I (facial and leptomeningeal angioma), type II (facial angioma without intracranial involvement) and type III (leptomeningeal angioma without facial nevus). Type III SWS is a rare variant. An atypical pattern of SWS may be the involvement of infratentorial structures. This may be associated with the apparent absence of supratentorial pial enhancement and delayed visualization of pial angiomatosis until childhood. Rarely, the disease can be bilateral [2, 12]. Approximately one third of the patients who have SWS have ocular or orbital abnormalities such as choroid venous malformation, congenital glaucoma with enlargement of the globe (buphthalmos) and optic disc colobomas. Overgrowth of the underlying facial skeleton may result in facial asymmetry and dental malocclusion. There may be facial capillary venous malformations with subjacent lymphatic malformation. Associated juvenile ossifying fibroma of the maxillofacial region as an expansile fibroosseous bony lesion has been reported [13]. 
Fig. 1 Sturge-Weber syndrome: a coronal contrast T1-weighted image shows hemiatrophy of the left cerebral hemisphere with abnormal leptomeningeal enhancement in the left parieto-occipital region and enlarged left choroid plexus. b Axial T2-weighted image shows left cerebral hemiatrophy atrophy with hyperintense $\mathrm{T} 2$ signal within the ischemic tissue and enlarged hyperintense left choroids plexus. c, d Contrast CT scan in another patient shows left temporal, parietal and occipital cerebral atrophy and abnormal leptomeningeal enhancement with underlying cortical calcifications
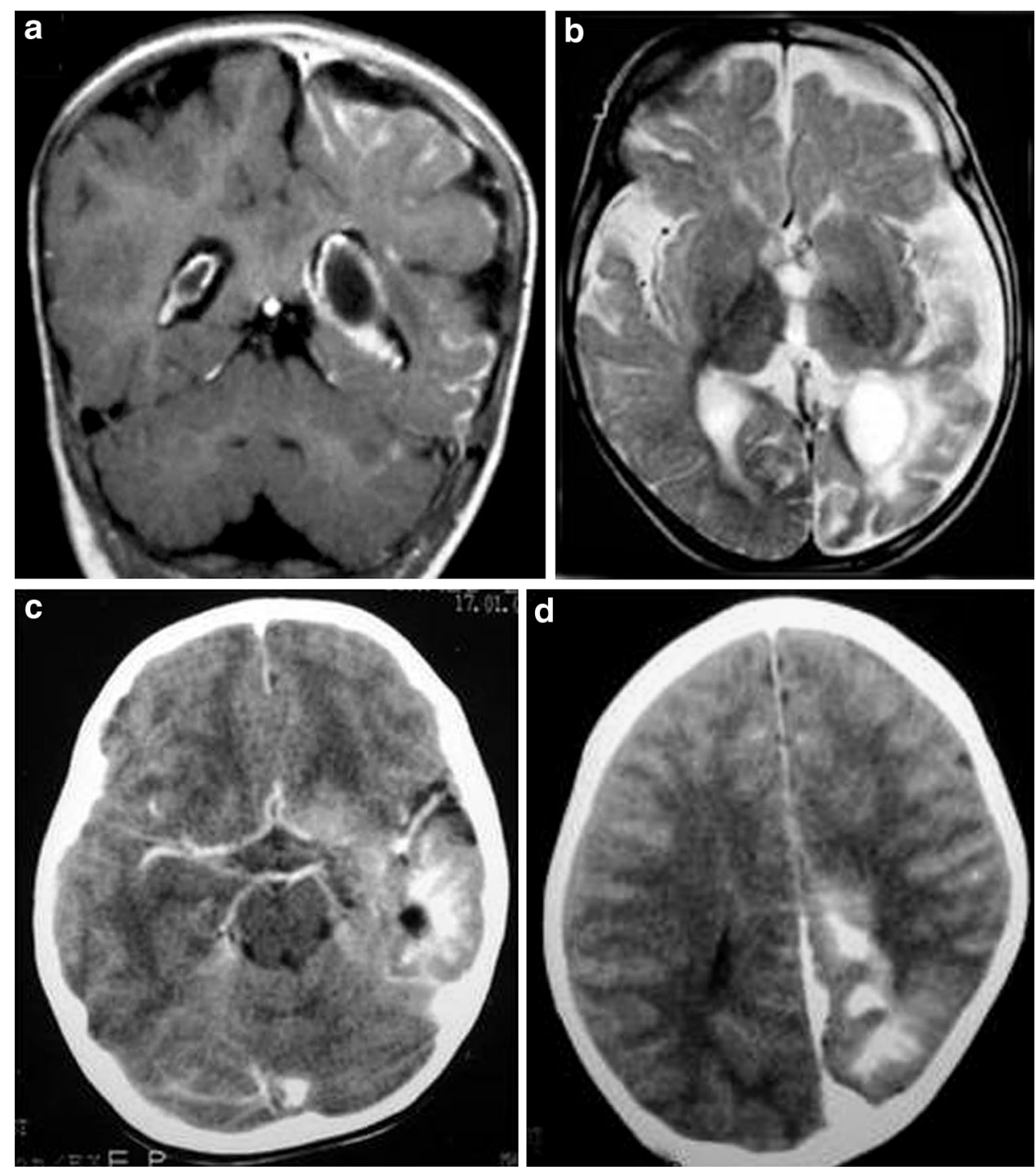

\section{PHACE syndrome}

The acronym PHACE describes the features of posterior fossa malformations, large facial hemangiomas, arterial anomalies, coarctation of the aorta and cardiac defects, and eye abnormalities. PHACE syndrome presents in $2 \%$ of children with facial hemangiomas and $20 \%$ with "segmental" facial hemangiomas. Approximately $90 \%$ of PHACE syndrome patients are female. PHACE syndrome may represent a field defect due to an insult in early embryonic development [6]. PHACE syndrome presents at birth with a large, plaque-like, ulcerated facial hemangioma along division V1 (75\%) and Dandy-Walker malformation $(30-80 \%)$. Less common are hypoplasia or agenesis of the cerebellum, vermis, and corpus callosum, abnormalities of the septum pellucidum and intracranial arachnoid cysts. Intracranial hemangiomas have a predilection for the cerebellopontine angle [14-16].
Posterior fossa malformations are present in 32-74\% of patients and represented by Dandy-Walker malformations and cerebellar hypoplasia (homolateral to the facial hemangioma). Of all Dandy-Walker malformations, $10 \%$ are associated with cutaneous hemangiomas [14-16]. Facial hemangiomas can be focal (tumor-like lesions) or diffuse (plaque-like lesions with a segmental pattern) (Fig. 2). Diffuse lesions affect the mandibular (38\%), maxillary (35\%) and fronto-nasal (27\%) segments; however, in $24 \%$ of cases, more than one facial segment is involved. It may be bilateral $(25 \%)$. Cerebrovascular anomalies associated with PHACES are hypoplasia or agenesis of the major cerebral vessels, persistence of embryonic vessels, arterial stenosis or occlusion, and dolichoectasia of the cerebral vasculature. Persistence of embryonic arteries, such as the persistent trigeminal artery, occurs most commonly, followed by partial or complete agenesis of major cervical arteries. Segmental agenesis of the internal carotid artery occurs with flow reconstruction through embryonic 


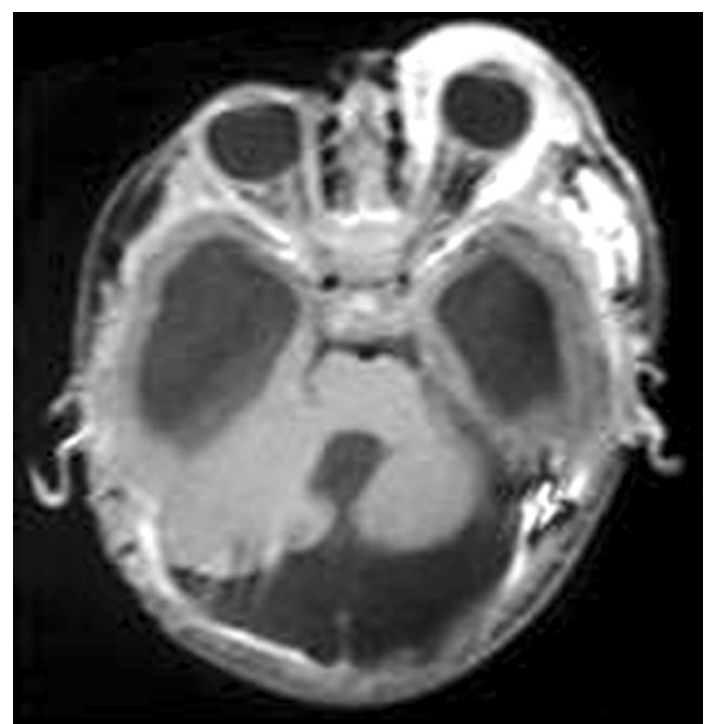

Fig. 2 PHACE: axial contrast T1-weighted image shows left orbital hemangioma demonstrating intense contrast enhancement associated with cerebellar hypoplasia in a child with PHACE syndrome

vessel anastomoses. Concerning stenoses, the onset of occlusive disease takes place between birth and 18 months of age, leading to pediatric strokes and a moyamoya-like appearance of the distal internal carotid artery [15]. Ocular abnormalities in the form of colobomas, optic nerve hypoplasia, increased retinal vascularity and glaucoma have been described [6]. Cardiac abnormalities are seen in one third of cases, with coarctation being the most common abnormality. Other cardiac anomalies include right-sided aorta, persistent ductus arteriosus, ventriculo-septal defects and pulmonary stenosis. The side of the hemangioma is ipsilateral to the side of the aortic arch abnormality [14-16].

\section{Craniofacial arteriovenous metameric syndrome}

Craniofacial arteriovenous metameric syndrome includes port-wine stains in one or several facial segments related to the migration of vascular cells from the mesoderm and neural crest, lymphangiomatous malformation of the cheek area, maxillofacial (malar, frontal or maxilla) and skull base (ethmoid, sphenoid petrous) hypertrophy and pial cortical venous occlusions (supra- or infratentorial) with associated gyral calcifications and atrophy. CAMS may involve two or three consecutive metamers and may be more or less complete in all tissues derived at a given level. Different groups of CAMS can be encountered: the medial prosencephalic group (olfactory) with involvement of the forehead and nose (CAMS 1); the lateral prosencephalic group (optic), with involvement of the occipital lobe, eye, cheek and maxilla (CAMS 2); and the rhombencephalon (otic) group with involvement of the cerebellum, lower face and mandible (CAMS 3) [17, 18] (Fig. 3).

\section{Wyburn-Mason syndrome (WMS)}

Wyburn-Mason syndrome is characterized by the triad of unilateral facial nevi, orbital AVMs and cerebral AVM along the visual pathway such as the optic chiasma, basal ganglion, midbrain and occipital cortex [1]. Bilateral cases are rarely reported. The patients typically present before the 3 rd decade. It belongs to the CAMS 2 originating from the lateral prosencephalon [18]. The disorder is a result of a developmental abnormality affecting the primitive vascular mesoderm shared by the developing optic cup and anterior neural tube [19]. In a review of 203 patients with brain vascular malformations, $9 \%$ of patients could be included in the multiple AVMs category, and only $1(0.4 \%)$ was considered to have WMS [20]. Imaging reveals high-flow vascular malformation within the orbits, retro-orbital region, cerebral hemispheres and brainstem, which may be associated with subarachnoid or intraparenchymal hemorrhage. Arterial supply to the AVMs usually arises from the internal carotid artery, and the venous drainage is through the cavernous sinus or vein of Galen. Rarely, AVM of the face, pharynx and palate and thyroid has been reported [1, 18-20].

\section{Spinal arteriovenous metameric syndrome}

Spinal arteriovenous metameric syndrome (Cobb syndrome) (cutaneomeningospinal angiomatosis) is a rare non-familial condition characterized by spinal AVMs with an overlying congenital cutaneous AVM in the same dermatome. Peak age is the 3rd and 5th decades. There is a slight male predominance. Lesions are frequently seen in the cervical and thoracic spinal cord. The embryological origin of the blood supply to the vertebra and spinal cord derives from the segmental dorsal arteries. The neurological manifestations are due to blood steal syndrome with cord ischemia or cord compression and venous hypertension. MR-revealed spinal cord edema probably secondary to venous stasis results in anterior spinal artery pressure and abnormal tissue drainage. Angiography shows spinal angiomas have a distinct blood supply from the normal spinal cord. They may be associated with lymphangioma circumscriptum, kyphoscoliosis and infantile uterus [2, 21] (Fig. 4).

\section{Hereditary hemorrhagic telangiectasia (Osler-Weber syndrome)}

Hereditary hemorrhagic telangiectasia, also known as RenduOsler-Weber syndrome, is an autosomal dominant vascular neurocutaneous disorder. The diagnostic criteria based on four Curaçao criteria of spontaneous recurrent nose bleeds, mucocutaneous telangiectasia at characteristic sites (the lips, 

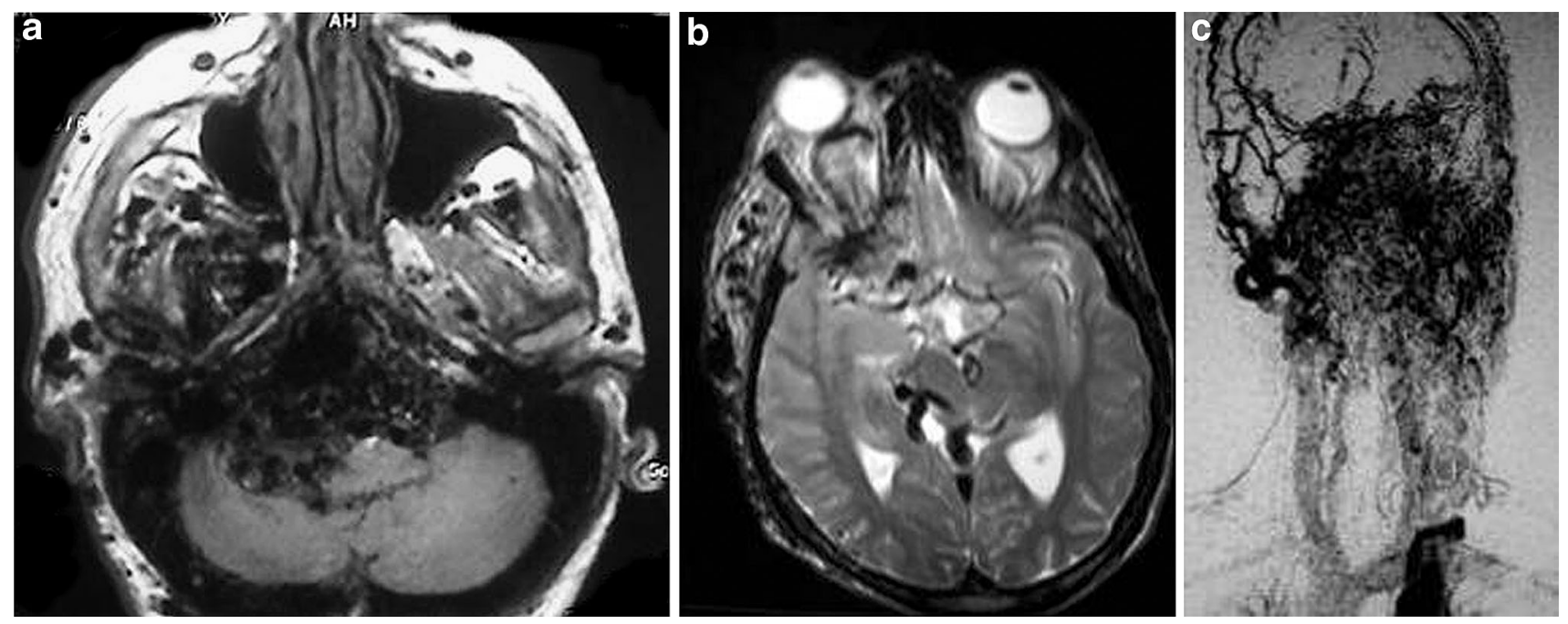

Fig. 3 Craniofacial arteriovenous metameric syndrome: a axial T2weighted image shows multiple signal-void regions of high-flow vascular malformations in the right masticator and parapharyngeal space as well as in the skull base and right external ear. b Axial T2-

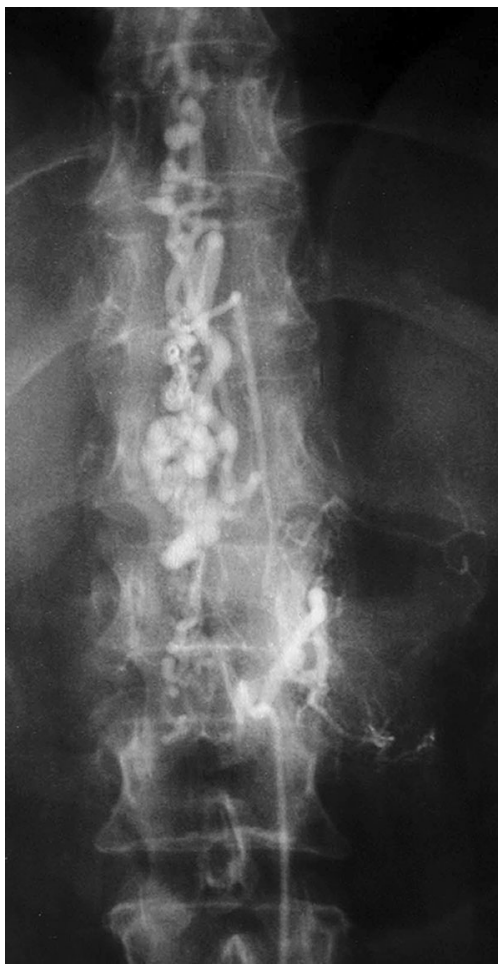

Fig. 4 Spinal arteriovenous metameric syndrome: conventional spinal angiography shows dilated tortuous high-flow spinal vascular malformation of the dorsal spine

oral cavity, fingers or nose), visceral involvement such as pulmonary, hepatic or CNS AVMs, and an affected firstdegree relative [2-4]. Patients present at the 4th-6th decades of life. HHT accounts for up to $60 \%$ of all pulmonary AVMs. Five percent to $27 \%$ of HHT patients have pulmonary weighted image at another level shows a signal void in the right facial subcutaneous tissue, right orbit and right intracranial region. c Contrast-enhanced MR angiography shows multiple dilated tortuous vascular structures of the AVM in the craniofacial region

AVMs. Hepatic involvement is often occult. Complex hepatic vascular alterations are seen in up to $74 \%$ of HHT patients, including arterioportal and arteriosystemic shunts, telangiectasias and vascular anatomic variants [22].

Neurological symptoms are reported in $8-27 \%$ of patients. The neurological deficits result from embolic complications of pulmonary AVMs in two thirds and intracerebral hemorrhage in one third. The mechanisms of CNS involvement in HHT are distal emboli, cerebrovascular malformation and metabolic disorders. Septic emboli HHT can cause arterial strokes in children. Most of them are embolic in nature and may be septic or aseptic. Usually, they are due to a pulmonary arteriovenous fistula (AVF). Multiple ischemic strokes in different arterial territories at different ages characterize the embolic mechanism in HHT. Brain abscesses (5-10\%) are usually multiple and recurrent, affecting the superficial layers of the cerebral lobes (mostly the parietal lobe) and developing at the 3rd-5th decades. Cerebral vascular malformations appear as areas of serpiginous flow voids insinuating into the brain parenchyma (Fig. 5). For metabolic disorder, T1-weighted images show hyperintensity of the basal nuclei related to hepatic portal vein shunt. Spinal AVM is reported in the dorso-lumbar region [22, 23]. Patients with HHT commonly present with epistaxis because of the highflow vascular malformation in the nose (Fig. 5).

\section{Blue rubber bleb nevus syndrome}

Blue rubber bleb nevus syndrome (Bean syndrome) is a rare vascular neurocutaneous disorder characterized by multiple vascular malformations of the skin and intestines 
Fig. 5 Hemorrhagic hereditary telangiectasia: a contrast MR angiography shows high-flow AVM in the frontal region. b Conventional angiography in another patient with recurrent epistaxis shows high-flow vascular malformation in the anterior part of the nose
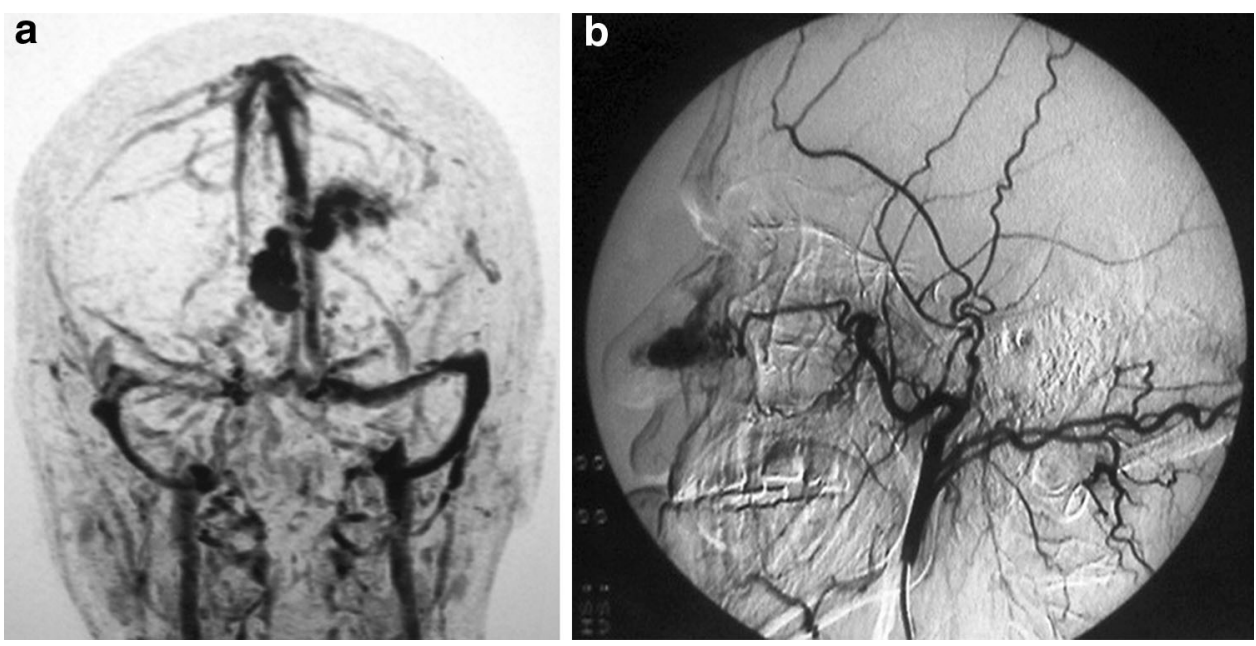
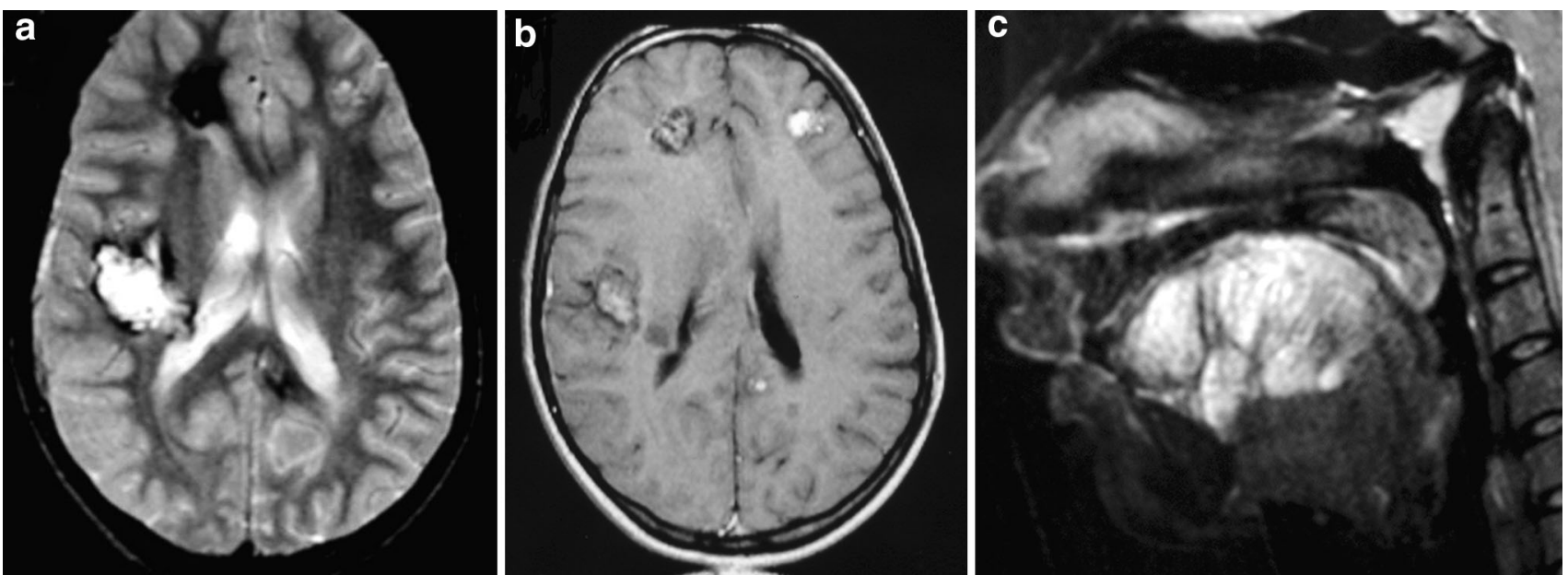

Fig. 6 Blue rubber bleb syndrome: a axial T2-weighted image shows multiple cavernomas in the supratentorial region. b Contrast MR imaging of the same patient shows an inhomogeneous pattern of contrast enhancement of these lesions. c Sagittal T2-weighted image

with gastrointestinal hemorrhage. Both genders are affected equally; most cases are diagnosed by 20 years of age. The incidence is lower in blacks. It is rarely reported in the CNS, orbit and thyroid. It characterized with multiple distinctive cutaneous and gastrointestinal venous malformations. Cutaneous lesions may number from a few to more than 100 [24-26].

The features of BRBNS of the brain consisted of multiple venous malformations, cavernous malformations and dural AVFs in supratentorial brain, cerebellum and diencephalon. Intracranial DAVFs are arteriovenous shunts imbedded in the dura mater and consist of abnormal direct communications from a dural arterial supply to a dural venous drainage. Cerebral cavernous malformations are vascular malformations characterized by abnormally enlarged capillary cavities, mostly located in the brain and less frequently in the spinal in another patient with diffuse venous malformation of the tongue shows high signal intensity of the tongue muscle with signal void regions

cord. Other neurovascular anomalies include aberrant venous sinuses, sinus pericranii and vein of Galen. Orbital venous malformations can be multiple and bilateral. Head and neck venous malformations appear as enhancing lobulated structures and may contain dense phleboliths (Fig. 6). The vertebral bodies may show stippled honeycomb lesions characteristic of hemangioma. Concurrent epidural hemangiomas within the spinal canal are reported in some cases. The associated tumors include medulloblastoma and squamous cell carcinoma [24-27].

\section{Klippel-Trenaunay syndrome}

The classical triad of KTS consists of: (1) vascular malformations of the capillary, venous and lymphatic vessels, 
Fig. 7 Klippel-Trenunay syndrome: a coronal T1weighted image shows giant aneurysm in the suprasellar region with signal void. b MRA of the same patient shows good delineation of the aneurysm
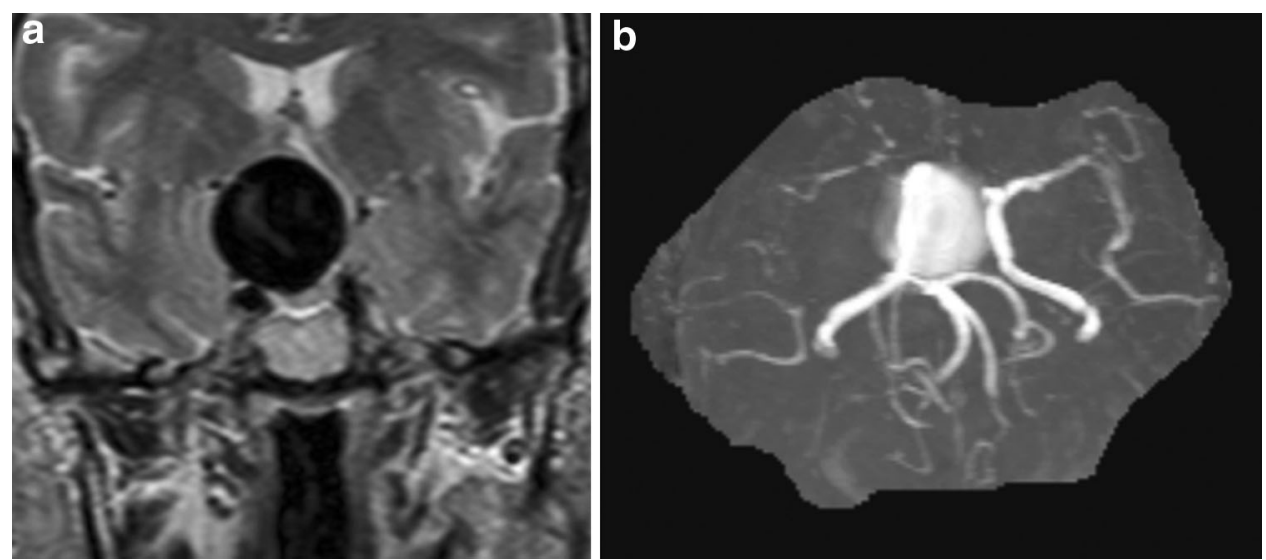

(2) varicosities of unusual distribution, particularly the lateral venous anomaly, and (3) unilateral limb enlargement, usually the lower extremity with hypertrophy of the bone and/or soft tissue and varicose veins. There are lowflow venous and lymphatic malformations of the soft tissue with anomalies of the superficial and deep venous system of the extremities. Gigantism involves both soft tissue and osseous structures and can affect the entire extremity. The most frequent CNS findings in patients with the KTS are changes in brain size, indicating hemimegalencephaly, or, less frequently, hemihypertrophy. KTS can be associated with aneurysms (Fig. 7), leptomeningeal vascular dysplasia and vascular malformations. Associated spinal cord vascular lesions are reported in some cases. Asymmetric growth of the craniofacial skeleton with increased facial dimensions of both the bone and soft tissue may lead to malformation of the jaws [28-30].

\section{Macrocephaly-capillary malformation}

Macrocephaly-capillary malformation is an overgrowth syndrome characterized by body asymmetry and cutaneous, vascular and neurological abnormalities. M-CM is assumed to have a genetic basis, which is still unknown, although it seems to occur sporadically. The diagnosis of $\mathrm{M}-\mathrm{CM}$ requires the presence of both of two major criteria, macrocephaly and capillary malformation, and at least two of the eight following minor criteria: asymmetry or overgrowth, developmental delay, midline facial capillary malformations, neonatal hypotonia, syndactyly/polydactyly, frontal bossing, joint hypermobility or hyperelastic skin, and hydrocephalus. At imaging, there is macrocephaly greater than the 95th percentile and capillary malformations. The structural abnormalities most frequently described are ventriculomegaly, hemimegalencephaly, white matter defects and Chiari type I malformations. Other findings include cavum septum pellucidum, cortical

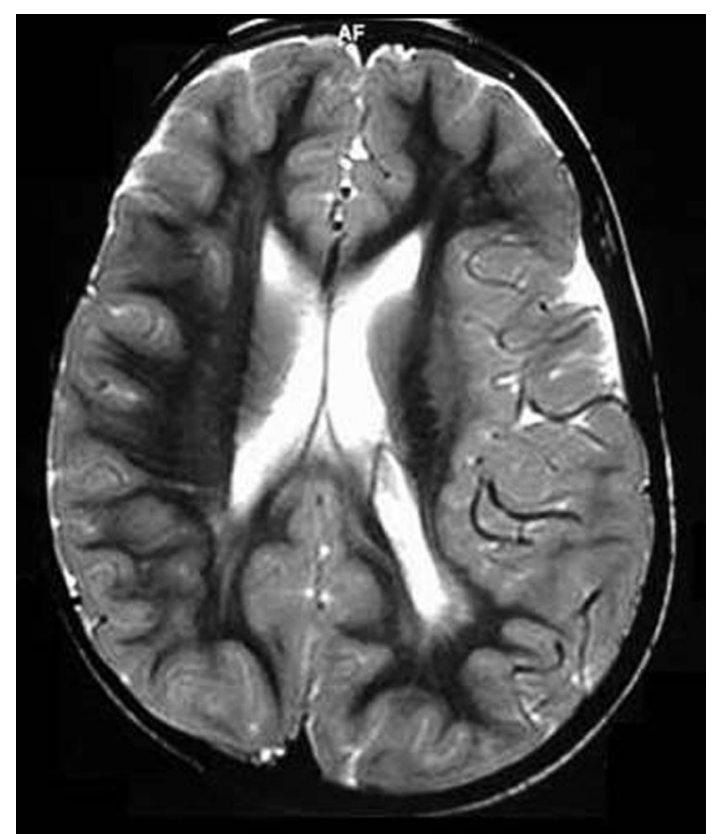

Fig. 8 Macrocephaly-capillary malformation: axial T2-weighted image shows mild cerebral asymmetry with focal enlargement of the left temporoparietal region. The enlarged region shows an abnormal gyral pattern

dysplasia, polymicrogyria, thick corpus callosum and hypoplastic cerebellum (Fig. 8). Cardiac abnormalities appear to be rare and include arrhythmia and complex congenital heart disease [31,32].

\section{Capillary malformation-arteriovenous malformation}

RASopathies include a set of common syndromes that have mutations on the Ras/MAPK pathway (RASAl gene). CM$\mathrm{AVM}$ is an autosomal dominant condition in which multifocal capillary malformations and arteriovenous fistulas form in different organs. The penetrance and de novo 


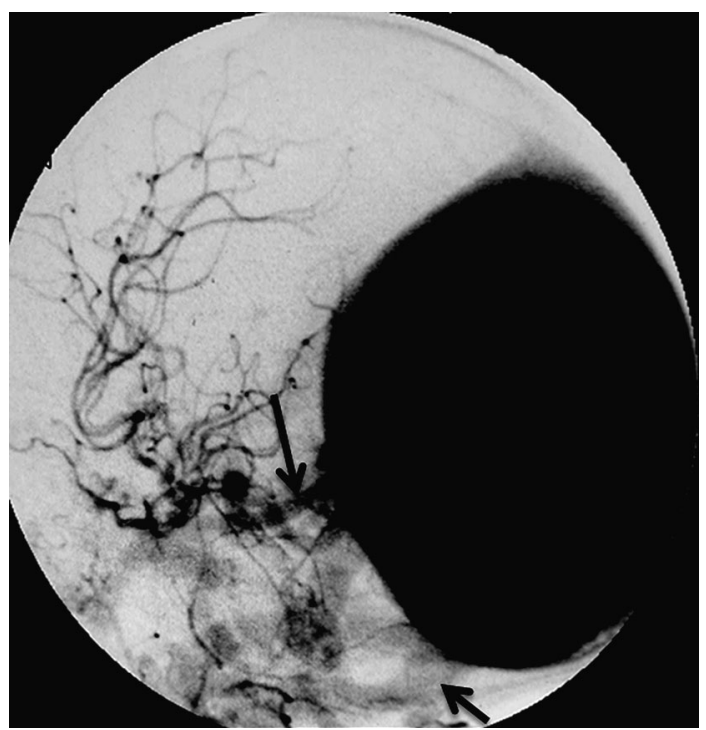

Fig. 9 Capillary malformation-arteriovenous malformation: lateral projection of late phase conventional angiography shows vein of Galen malformation. The arterial vascularization of malformation is through choroidal systems (long arrow) and the drainage is finally through the transverse sinus (short arrow)

occurrence are high. Capillary malformation, presenting as a red macular stain that can darken over years, is a common birthmark. The associated high-flow anomalies include $\mathrm{AVM}$ and $\mathrm{AVF}$, including vein of Galen malformation (Fig. 9) in the brain (7.1\%), face (7.8\%) or limbs (3.6\%). Intracranial arteriovenous lesions are typically macrofistulous, usually presenting with neurological signs at birth or before 1 year of age. Small, usually multifocal and randomly distributed cutaneous capillary malformations are the most prominent feature in patients with RASAl mutations [33, 34].

\section{Meningioangiomatosis}

Meningioangiomatosis is a rare, focal benign hamartomatous cortical or subcortical mass that may extend to the overlying leptomeninges forming a plaque-like lesion [1]. Two types of MA are broadly accepted: sporadic MA and neurofibromatosis type 2 (NF2) associated MA. Sporadic lesions are thought to be four times more common than MA with NF. Sporadic MA usually presents with medically intractable seizures in children or young adults, and NF2-associated MAs are usually asymptomatic. The cerebral cortex and subcortical white matter are concomitantly involved by MA. Subcortical white matter components of MA are more apparent than cortical components. Spotty or patchy calcification of the lesion and hypertrophy of the cortex on CT scan are important features of MA. In MRI findings, MA usually appears as a small solid mass located

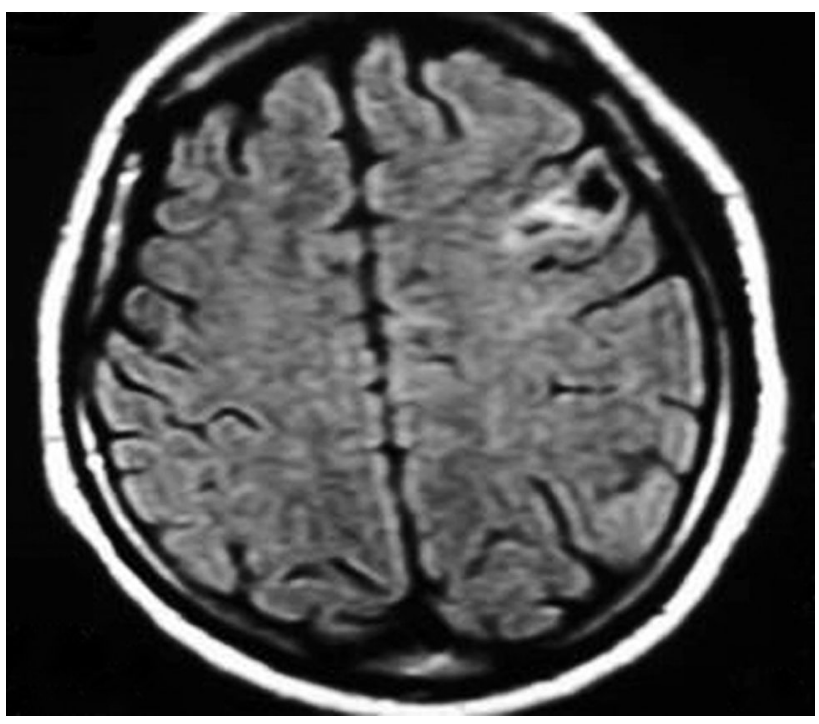

Fig. 10 Meningioangiomatosis: axial FLAIR image shows cortical and subcortical lesions in the left parietal region that exhibit low signal intensity with marginal hyperintensity

beneath the superficial cortex, presenting an enlarged gyrilike formation. On T1WI, the lesions often show iso- or hypointensity compared to the gray matter. This tends to make the lesion invisible at a glance. On T2WI and FLAIR images, the signals are heterogeneous, typically mainly hypointense alternated with iso-/hyperintensity, making the lesion more clear cut than on T1WI. In some circumstances, there is surrounding hyperintensity, which could be explained as edema or gliosis. However, sometimes the hyperintensity may refer to CSF in the regional subarachnoid space, which can be totally suppressed by FLAIR imaging rather than part of the lesion (Fig. 10). Most lesions partially demonstrate very slight spotty enhancement or do not enhance at all [35-37].

\section{Ataxia-telangiectasia (A-T) (Louis Bar syndrome)}

Ataxia-telangiectasia is an autosomal recessive multisystem disease of childhood characterized by progressive cerebellar degeneration and ataxia that begins between 1 and 3 years of age, oculocutaneous telangiectasias, and increased susceptibility to infection and malignancies. The gene responsible for A-T, ataxia telangiectasia mutated (ATM), is located on chromosome 11q22-23. The cerebellum shows atrophy of all cortical layers and the dentate nuclei. The severe loss of Purkinje cells affects the vermis, cerebellar hemispheres or both. Telangiectatic vessels are seen in the leptomeninges in A-T [38]. Patients with A-T show marked atrophy of the vermis, cerebellar hemispheres and denate nuclei with prominent foci and dilation of the 


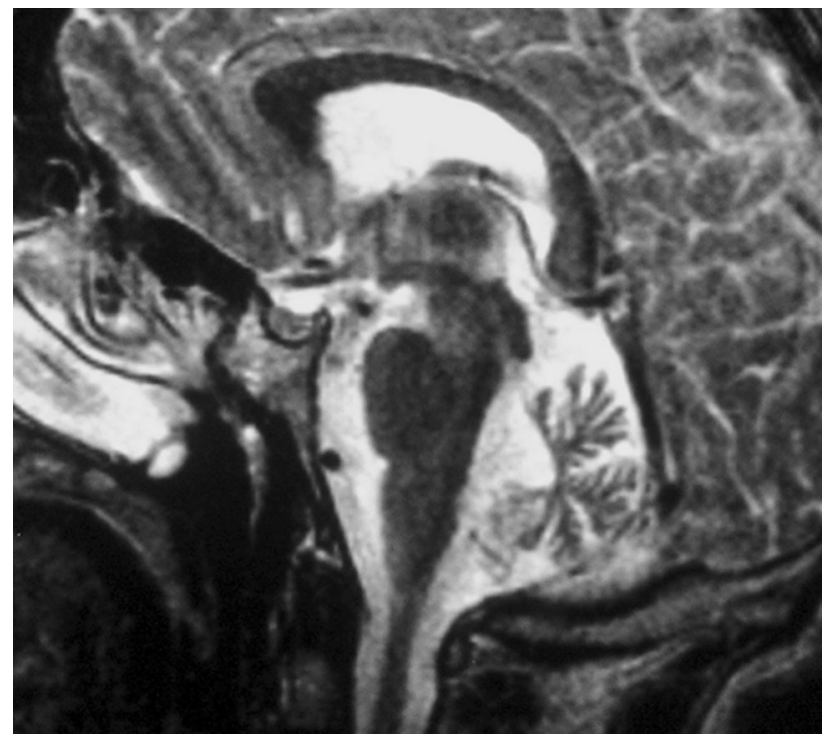

Fig. 11 Ataxia-telangiectasia: sagittal T2-weighted image shows cortical thinning and fissure enlargement in the vermis and cerebellar hemispheres but normal volume of the brain stem

fourth ventricle (Fig. 11). The atrophy is panvermian and progressive. There is an increase in choline on MR spectroscopy in contrast to other ataxias. Supratentorial brain abnormalities are reported very rarely. Intracerebral telangiectasia with multiple punctate hemosiderin deposits is reported in $60 \%$ of subjects. These lesions were apparently asymptomatic. Extensive white matter T2 hyperintensity has been reported [39]. A spectrum of paranasal sinus inflammatory disease due to immunodeficiency has been reported. Patients also have a predisposition to malignancies of which the predominant types are lymphomas and lymphoid leukemias as well as neural tumors such as medulloblastoma and glioma [38, 39].

\section{Conclusion}

We concluded that segmental or localized vascular neurocutaneous disorders are characterized by the presence of low- or high-flow vascular malformations that may be associated with abnormal growth such as hemimegalencephaly and different craniofacial or spinal anomalies. Hamartomatous formation can be found in different tissues or associated with malignancy. Correlation with clinical findings of neurospinal and craniofacial abnormalities is important to reach a specific diagnosis for some vascular neurocutaneous disorders.

Conflict of interest The author declares no conflict of interest.

\section{References}

1. Edelstein $S$, Naidich $T$, Newton $T$. The rare phakomatoses. Neuroimaging Clin N Am. 2004;14:185-217.

2. Pascual-Castroviejo I, Di Rocco C, editors. Neurocutaneous disorders phakomatoses and hamartoneoplastic syndromes. Wien: Springer; 2008.

3. Nandigam K, Mechtler L, Smirniotopoulos J. Neuroimaging of neurocutaneous diseases. Neurol Clin. 2014;32:159-92.

4. Vanaman MJ, Hervey-Jumper SL, Maher CO. Pediatric and inherited neurovascular diseases. Neurosurg Clin $\mathrm{N}$ Am. 2010;21:427-41.

5. Elsayes KM, Menias CO, Dillman JR, Platt JF, Willatt JM, Heiken JP. Vascular malformation and hemangiomatosis syndromes: spectrum of imaging manifestations. AJR Am J Roentgenol. 2008;190:1291-9.

6. Krings T, Geibprasert S, Luo CB, Bhattacharya JJ, Alvarez H, Lasjaunias P. Segmental neurovascular syndromes in children. Neuroimaging Clin N Am. 2007;17:245-58.

7. Nozaki T, Nosaka S, Miyazaki O, Makidono A, Yamamoto A, Niwa T, et al. Syndromes associated with vascular tumors and malformations: a pictorial review. Radiographics. 2013;33:175-95.

8. Abdel Razek A, Kandell A, Elsorogy L, Elmongy A, Abdel basett A. Disorders of cortical formation: MR imaging features. Am J Neuroradiol (AJNR). 2009;30:4-11.

9. Razek AA, Gaballa G, Megahed A, Elmogy S. Time resolved imaging of contrast kinetics (TRICKS) MR angiography of arteriovenous malformations of head and neck. Eur J Radiol. 2013;82:1885-91.

10. Abdel Razek A, Denewer A, Hegazy M, Hafez M. Role of CT angiography in diagnosis of vascular stenosis of head and neck microvascular free flap reconstruction. Int J Maxillofac Surg. 2014;43:811-5.

11. Juhasz C, Haacke EM, Hu J, Xuan Y, Makki M, Behen ME, et al. Multimodality imaging of cortical and white matter abnormalities in Sturge-Weber syndrome. AJNR Am J Neuroradiol. 2007;28:900-6.

12. Adams ME, Aylett SE, Squier W, Chong W. A spectrum of unusual neuroimaging findings in patients with suspected SturgeWeber syndrome. AJNR Am J Neuroradiol. 2009;30:276-81.

13. Lin D, Gailloud P, McCarthy E, Comi A. Oromaxillofacial osseous abnormality in Sturge-Weber syndrome: case report and review of the literature. AJNR Am J Neuroradiol. 2006;27:274-7.

14. Oza V, Wang E, Berenstein A, Waner M, Lefton D, Wells J, et al. PHACES association: a neuroradiologic review of 17 patients. AJNR Am J Neuroradiol. 2008;29:807-13.

15. Judd CD, Chapman PR, Koch B, Shea CJ. Intracranial infantile hemangiomas associated with PHACE syndrome. AJNR Am J Neuroradiol. 2007;28:25-9.

16. Church D, Lowe L. PHACE syndrome. Radiology. 2006;241:939-42.

17. Bun YY, Ming CK, Ming CH, Ling CY, Ming CC. Endovascular treatment of a neonate with dural arteriovenous fistula and other features suggestive of cerebrofacial arteriovenous metameric syndromes. Childs Nerv Syst. 2009;25:383-7.

18. Wong IY, Batista LL, Alvarez H, Lasjaunias PL. Craniofacial arteriovenous metameric syndrome (CAMS) 3-a transitional pattern between CAM 1 and 2 and spinal arteriovenous metameric syndromes. Neuroradiology. 2003;45:611-5.

19. Dayani P, Sadun A. A case report of Wyburn-Mason syndrome and review of the literature. Neuroradiology. 2007;49:445-56.

20. Willinsky R, Lasjaunias P, terBrugge K, Montanera W. Multiple cerebral arteriovenous malformations: review of our experience 
from 203 patients with cerebral vascular lesions. Neuroradiology. 1990;32:207-10.

21. Johnson W, Petrie M. Variety of spinal vascular pathology seen in adult Cobb syndrome. Report of 2 cases. J Neurosurg Spine. 2009;10:430-5.

22. Carette M, Nedelcu C, Tassart M, Grange J, Wislez M, Khalil A. Imaging of hereditary hemorrhagic telangiectasia. Cardiovasc Interv Radiol. 2009;32:745-57.

23. Krings T, Ozanne A, Chng S, Alvarez H, Rodesch G, Lasjaunias P. Hereditary hemorrhagic telangiectasia neurovascular phenotypes and endovascular treatment. Clin Neuroradiol. 2006;16:76-90.

24. Carvalho S, Barbosa V, Santos N, Machado E. Blue rubber-bleb nevus syndrome: report of a familial case with a dural arteriovenous fistula. AJNR Am J Neuroradiol. 2003;24:1916-8.

25. Park CO, Park J, Chung KY. Blue rubber bleb nevus syndrome with central nervous system involvement. J Dermatol. 2006;33:649-51.

26. Starr BM, Katzenmeyer WK, Guinto F, Pou AM. The blue rubber bleb nevus syndrome: a case with prominent head and neck findings. Am J Otolaryngol. 2005;26:282-4.

27. Garen PD, Sahn EE. Spinal cord compression in blue rubber bleb nevus syndrome. Arch Dermatol. 1994;130:934-5.

28. Vurucu S, Battal B, Kocaoglu M, Akin R. Klippel-Trenaunay syndrome with hemimegalencephaly, retroperitoneal lymphangioma and double inferior vena cava. $\mathrm{Br} \mathrm{J}$ Radiol. 2009;82:e102-4.

29. Rohany M, Shaibani A, Arafat O, Walker M, Russell E, Batjer H, et al. Spinal arteriovenous malformations associated with Klippel-Trenaunay-Weber syndrome: a literature search and report of two cases. AJNR Am J Neuroradiol. 2007;28:584-9.

30. Defraia E, Baccetti T, Marinelli A, Tollaro I. Biometric and magnetic resonance imaging assessment of dentofacial abnormalities in a case of Klippel-Trenaunay-Weber syndrome. Oral Surg Oral Med Oral Pathol Oral Radiol Endod. 2004;97:127-32.
31. Papetti L, Tarani L, Nicita F, Ruggieri M, Mattiucci C, Mancini $\mathrm{F}$, et al. Macrocephaly-capillary malformation syndrome: description of a case and review of clinical diagnostic criteria. Brain Dev. 2012;34:143-7.

32. Martínez-Glez V, Romanelli V, Mori MA, Gracia R, Segovia M, González-Meneses A, et al. Macrocephaly-capillary malformation: analysis of 13 patients and review of the diagnostic criteria. Am J Med Genet A. 2010;152A:3101-6.

33. Thiex R, Mulliken J, Revencu N, Boon L, Burrows P, Cordisco $\mathrm{M}$, et al. A novel association between RASAl mutations and spinal arteriovenous anomalies. AJNR Am J Neuroradiol. 2010;31:775-9.

34. Eerola I, Boon LM, Mulliken JB, Burrows PE, Dompmartin A, Watanabe $S$, et al. Capillary malformation-arteriovenous malformation, a new clinical and genetic disorder caused by RASA1 mutations. Am J Hum Genet. 2003;73:1240-9.

35. Jeon TY, Kim JH, Suh YL, Ahn S, Yoo SY, Eo H. Sporadic meningioangiomatosis: imaging findings with histopathologic correlations in seven patients. Neuroradiology. 2013;55:1439-46.

36. Feng R, Hu J, Che X, Pan L, Wang Z, Zhang M, et al. Diagnosis and surgical treatment of sporadic meningioangiomatosis. Clin Neurol Neurosurg. 2013;115:1407-14.

37. Abdulazim A, Samis Zella MA, Rapp M, Gierga K, Langen KJ, Steiger HJ, et al. Meningioangiomatosis in a patient with progressive focal neurological deficit-case report and review of literature. Br J Neurosurg. 2013;27:253-5.

38. Wallis L, Griffiths P, Ritchie S, Romanowski C, Darwent C, Wilkinson I. Proton spectroscopy and imaging at 3T in ataxiatelangiectasia. AJNR Am J Neuroradiol. 2007;28:79-83.

39. Lin DD, Barker PB, Lederman HM, Crawford TO. Cerebral abnormalities in adults with ataxia-telangiectasia. AJNR Am J Neuroradiol. 2014;35:119-23. 